\title{
Comparative efficacy of six therapies for Hypopharyngeal and laryngeal neoplasms: a network meta-analysis
}

\author{
Juan Che, Yanlin Wang, Xiaolin Zhang and Jun Chen ${ }^{*}$
}

\begin{abstract}
Background: Hypopharyngeal and laryngeal neoplasms are both fatal and hard to catch in early stages. Yet which treatment is the most efficacious one still remain unanswered. This network meta-analysis (NMA) was conducted to investigate effectiveness of six therapies being utilized in clinical practice nowadays.

Methods: PubMed and Embase were retrieved to synthesize data. Direct and indirect evidence was combined to compare efficacy of treatments. A relative ranking of the six regimens was calculated by the surface under the curve ranking area (SUCRA).

Results: A total of 28 trials with 9109 patients were included in our NMA. Five endpoints investigated included 3/5year overall survival (3/5-OS), 3/5-year disease free survival (3/5-DFS) and 5-year overall survival rate (5-OSR). In terms of all efficacy outcomes, radiotherapy combined with surgery $(R T+S)$ proved to be better than other therapies while radiotherapy (RT) alone also performed well. Induction chemoradiotherapy (ICRT) was the best regarding 3-DFS (SUCRA $=0.846$ ) while current chemoradiotherapy (CCRT) ranked first in 5-DFS (SUCRA $=0.933$ ) according to SUCRA results. No significant differences were demonstrated in 5-DFS and 5-OSR as shown in the results of NMA.

Conclusions: RT combined with surgery turned out to be optimal therapy of all the outcomes while the efficacy of RT was relatively poorer in the treatment of patients with larynx stage III-IV and hypopharynx stage II-IV. Also, the good performance of CCRT and ICRT in terms of DFS made them as secondary recommended therapies. There is no significant difference between surgery and transoral laser microsurgery (TLM) alone.
\end{abstract}

Keywords: Hypopharyngeal neoplasm, Laryngeal neoplasm, Efficacy, Network meta-analysis

\section{Background}

Hypopharyngeal neoplasm, as a term used for malignancies of a subsite of the upper aerodigestive tract, is difficult to catch in its earliest stages. Meanwhile it is reported to have the highest mortality rates among kinds of head and neck cancers [1]. Laryngeal neoplasms are mostly squamous cell carcinomas, which reflect they originate from the skin of the larynx. Its 5 years survival rates in the United States are 60\% [2]. So far multiple treatments for hypopharyngeal or laryngeal neoplasms have been developed and compared, which include surgery, radiation therapy (RT), chemoradiation therapy (CRT), or kinds of combination of treatments aforementioned [3].

\footnotetext{
* Correspondence: diandianxing1979@163.com

Department of Otorhinolaryngology, Binzhou Medical University Hospital, No. 661 Second Huanghe Road, Binzhou 256603, Shandong, China
}

One of the main targets of treating patients with locoregional advanced laryngeal cancer is to maintain larynx function. Yet surgery, though providing satisfying local control, might impair life quality of patients. Thus the application of surgery alone in treating laryngeal neoplasms and hypopharyngeal neoplasms has been reduced while chemotherapy (CT) and RT have been more widely used in clinical practice these days [2]. However, RT and CT being applied separately or combined have triggered worries regarding a decrease in overall survival rate (OSR) since they are reckoned to have caused acute toxicity and disrupted laryngeal function [4].

Many meta-analyses related to head and neck cancer, hypopharyngeal neoplasm, and laryngeal neoplasms have been conducted to evaluate the efficacy of CT, RT, and CRT. Whereas, there were several deficiencies in prior

(C) The Author(s). 2019 Open Access This article is distributed under the terms of the Creative Commons Attribution 4.0 International License (http://creativecommons.org/licenses/by/4.0/), which permits unrestricted use, distribution, and 
studies such as small sample sizes [5] or a lack of sufficient prospective studies [5]. Besides, the nature of conventional meta-analysis prevents it from performing more comprehensive analysis of multiple treatments. It is known that network meta-analysis (NMA) could combine both direct and indirect evidence, thus considered to be of high clinical value. Based on the facts mentioned above, NMA might be the most adequate way to compare different regimens of hypopharyngeal neoplasms and laryngeal neoplasms.

Notably, most of existing NMAs comparing treatments for head and neck cancers failed to pay attention to certain specific types of cancers, which are hypopharyngeal and laryngeal neoplasms in our case. Hence it is necessary to conduct a systematic review in order to get more comprehensive results regarding regimens for hypopharyngeal and laryngeal neoplasms.

\section{Methods}

\section{Research reporting format}

This NMA was conducted according to the preferred reporting items for systematic reviews and meta-analyses (PRISMA) guidelines.

\section{Search strategy}

PubMed and Embase were systematically retrieved for eligible trials. Subject terms and key words including "hypopharyngeal neoplasms", "laryngeal neoplasms", "radiotherapy", "chemoradiotherapy", "transoral laser microsurgery", "radiotherapy combined with surgery", "induction chemotherapy radiotherapy", "current chemotherapy radiotherapy", and "clinical controlled trials" were searched with Boolean operators OR and AND. Titles and abstracts of all studies obtained after searching were reviewed in turn to screen their qualifications.

\section{Inclusion and exclusion criteria}

Included studies were restricted by the following criteria: (1) the study was clinical controlled trial; (2) patients were diagnosed with hypopharyngeal neoplasms or laryngeal neoplasms; (3) therapies included at least one of the six following treatments: surgery, RT, transoral laser microsurgery (TLM), radiotherapy combined with surgery $(\mathrm{RT}+\mathrm{S})$, induction chemotherapy radiotherapy (ICRT), and current chemotherapy radiotherapy (CCRT); (4) at least one of the following relevant outcomes was provided: $3 / 5$-year overall survival (3/5-OS), 3/5-year disease free survival (3/5-DFS) and 5-year overall survival rate (5-OSR). Conference abstracts, reviews, duplicates and articles lacking sufficient information or relevant outcomes were excluded. Two experienced reviewers performed an assessment independently to examine eligibility. Any possible disagreements between them would be resolved by consensus and still remaining disagreements would be referred to a third reviewer.

\section{Outcome measurement and data extraction}

Baseline characteristics of patients and basic information including first author, year of publication, blind test, etiology, follow-up duration, intervention, and sample size of each treatment were extracted by two reviewers from eligible trials. Discrepancies were solved by discussion or a third reviewer. Five outcomes were selected in order to assess the efficacy of surgery and other five regimens.

\section{Statistical analysis}

This NMA is conducted by $\mathrm{R}$ software (Version 3.1.3) and STATA (Version 13.0). A random-effect Bayesian model was utilized considering the existence of heterogeneity. Network plots demonstrated all efficacy outcomes of six regimens. Hazard ratios (HR) with 95\% credible intervals (CrIs) were calculated to perform a NMA, combining both direct and indirect evidence. The results of NMA were shown in forest plots and heat plots were utilized in our NMA to evaluate the inconsistency, in which colors were associated with the change in inconsistency between direct and indirect evidence (shown in the row) after detaching the effect of certain evidence (shown in the column). In heat plots, cold colors indicated an increase of consistency while warm colors indicated a decrease of consistency. In addition, relative efficacy of six therapies was ranked based on surface under the cumulative ranking curve (SUCRA). Higher probabilities revealed in SUCRA signified more desirable interventions.

\section{Subgroup analysis}

A subgroup analysis for locally advanced hypopharyngeal neoplasms or laryngeal neoplasms patients were conducted for the existence of clinical heterogeneity between studies. The subgroup analysis involved 17 studies reported patients with larynx stage III-IV and hypopharynx stage II-IV only.

\section{Results}

\section{Literature search}

A total of 4737 potential relevant publications were identified by literature search. Four thousand five hundred eighty-three remained for screening after duplicates were deleted. Four thousand three hundred forty-eight articles were excluded due to irrelevant titles and abstracts. Among the 235 full-text studies being assessed, 207 papers were removed on account of insufficient data, single arm studies, and irrelevant outcomes. 
Finally, 28 trials met the inclusion criteria and were retrieved for data extraction [2, 3, 6-31].

Table 1 and Additional file 1: Table S1 summarized basic information of included trials and patients characteristics. Among 28 trials with 9109 patients, most were two-arm trials comparing one kind of treatment with surgery or a surgery combined with other regimens. Among the included studies, there were seven trials designed to be randomized controlled trials while 21 were reported as retrospective cohort studies. The networks of comparisons between different treatments with corresponding sample sizes were summarized in Fig. 1. Each circle indicated a certain intervention while the size of each circle denoted its sample size. The thickness of lines linking two circles signified the amount of literatures comparing the corresponding two therapies.

\section{Outcomes}

In this NMA, 3-OS, 5-OS, 3-DFS, 5-DFS, and 5-OSR were calculated as primary outcomes, the results of which were demonstrated in Table 2 and Fig. 2.

In terms of 3-OS, surgery alone performed significantly better than RT and CCRT (RT: HR 1.25, 95\% CrI 1.00-1.57; CCRT: HR 1.23, 95\% CrI 1.00-1.53). Meanwhile, RT combined with surgery turned out to be significantly superior to RT, ICRT, and CCRT (RT: HR 1.31, 95\% CrI 1.06-1.61; ICRT: HR 1.22, 95\% CrI 1.021.47; CCRT: HR 1.28, 95\% CrI 1.06-1.55). With regard to 5-OS, RT combined with surgery and surgery alone both had significant advantage over RT (HR 1.25, 95\% CrI 1.01-1.55; HR 1.27, 95\% CrI 1.01-1.59, respectively). Apart from that, there existed no other significant difference among comparisons of 6 regimens.

Table 1 Basic characteristics of included studies

\begin{tabular}{|c|c|c|c|c|c|}
\hline Study & Design & Cancer type & Stage & No. of patients & Comparison \\
\hline Wolf, 2017 & Retrospective & Glottic/supraglottic cancer & I-IV & 247 & RT vs. CCRT vs. S \\
\hline Stokes, 2017 & Retrospective & Larynx cancer & IV & 3542 & $R T+S$ vs. CCRT vs. ICRT \\
\hline Low, 2017 & Retrospective & Laryngeal SCC & I & 105 & TLM vs. RT \\
\hline Peng, 2016 & Retrospective & Glottic cancer & $|-| \mid$ & 172 & RT vs. S \\
\hline Marchiano, 2016 & Retrospective & Subglottic SCC & I-IV & 576 & RT vs. S vs. RT + S \\
\hline De Santis, 2016 & Retrospective & Glottic cancer & $|-| \mid$ & 75 & TLM vs. RT \\
\hline Timmermans, 2015 & Retrospective & Laryngeal cancer & III-IV & 182 & S vs. CCRT vs. RT \\
\hline Timme, 2015 & Retrospective & Laryngeal cancer & III-IV & 71 & S vs. CCRT \\
\hline Li, 2015 & Retrospective & Laryngeal cancer & I-IV & 309 & RT vs. S vs. RT + S \\
\hline Grover, 2015 & Retrospective & Larynx cancer & IV & 969 & S vs. CCRT \\
\hline Hsin, 2014 & Retrospective & Laryngeal cancer & IV & 62 & S vs. CCRT \\
\hline Lefebvre, 2013 & Randomized & Larynx/Hypopharynx SCC & II-IV & 118 & CCRT vs. ICRT \\
\hline Forastiere, 2013 & Randomized & Larynx cancer & III-IV & 520 & CCRT vs. ICRT vs. RT \\
\hline Lefebvre, 2012 & Randomized & Hypopharyngeal SCC & II-IV & 194 & $R T+S$ vs. ICRT \\
\hline Patel, 2011 & Retrospective & Larynx cancer & IV & 34 & CCRT vs. S \\
\hline Mahler, 2010 & Retrospective & Glottic cancer & I & 351 & RT vs. TLM \\
\hline Dinapoli, 2010 & Retrospective & Glottic cancer & $|-| \mid$ & 143 & RT vs. S \\
\hline Schrijvers, 2009 & Retrospective & Glottic laryngeal cancer & I & 100 & RT vs. TLM \\
\hline Thurnher, 2008 & Retrospective & Glottic laryngeal SCC & I & 337 & S vs. TLM vs. RT \\
\hline Boscolo-Rizzo, 2008 & Retrospective & Laryngeal cancer & III-IV & 112 & $R T+S$ vs. CCRT \\
\hline Andreadis, 2007 & Retrospective & Laryngeal cancer & III-IV & 50 & ICRT vs. CCRT \\
\hline Bensadoun, 2006 & Randomized & Laryngeal cancer & III-IV & 163 & RT vs. CCRT \\
\hline Richard, 1998 & Randomized & Laryngeal cancer & III-IV & 68 & $R T+S$ vs. ICRT \\
\hline Beauvillain, 1997 & Randomized & Hypopharyngeal cancer & III-IV & 90 & $R T+S$ vs. S \\
\hline Bryant, 1995 & Retrospective & Glottic cancer & III & 97 & RT vs. RT + S \\
\hline Frank, 1994 & Retrospective & Hypopharyngeal SCC & I-IV & 109 & $S$ vs. RT $+S$ \\
\hline Jones, 1992 & Retrospective & Larynx cancer & III-IV & 147 & S vs. RT + S \\
\hline Wolf, 1991 & Randomized & Larynx cancer & III-IV & 166 & ICRT vs. CCRT \\
\hline
\end{tabular}

Abbreviation: $S$ surgery, $R T$ radiotherapy, $T L M$ transoral laser microsurgery, $R T+S$ surgery combined with radiotherapy, ICRT Induction chemotherapy radiotherapy, CCRT, current chemotherapy radiotherapy 


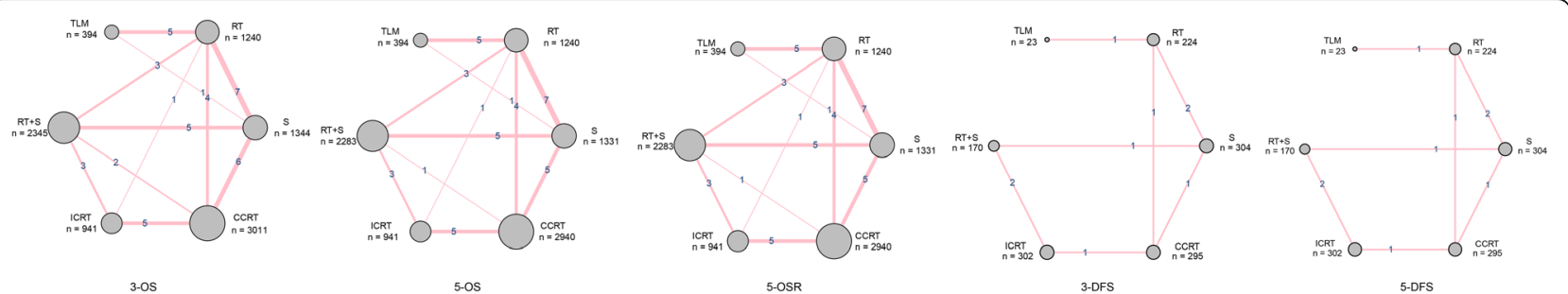

Fig. 1 The network of comparisons of 3-OS, 5-OS, 3-DFS, and 5-DFS. (The width of the lines is proportional to the number of trials comparing each pair of treatments; the area of circles represents the cumulative number of patients for each intervention.) S, surgery; RT, radiotherapy; TLM, transoral laser microsurgery; RT + S, surgery combined with radiotherapy; ICRT, Induction chemotherapy radiotherapy; CCRT, current chemotherapy radiotherapy

The results of 3-DFS were in favor of CCRT, since CCRT proved to be more efficacious than RT with statistical significance (HR 1.79, 95\% CrI 1.06-3.04). Yet no other significant difference occurred regarding 3-DFS. Notably, there also existed no significant difference among comparisons in 5-DFS and 5-OSR.

\section{Relative ranking analysis}

A relative ranking of the 6 therapies could be obtained on the basis of SUCRA results (Table 3). RT combined with surgery and surgery alone demonstrated desirable performance. RT combined with surgery and surgery alone ranked first and second respectively in both 3-OS $(\mathrm{RT}+\mathrm{S}:$ 0.878; S: 0.763$)$ and 5-OS (RT + S: 0.930; S: $0.773)$. Besides, surgery ranked highest in 5-ORS (0.716) while RT combined with surgery rank the third place in 3-DFS (0.717).

Moreover, ICRT and CCRT proved to be advantageous regarding DFS. ICRT was the optimal therapy in 3-DFS (0.846) and suboptimal in 5-DFS (0.639) while CCRT turned out to be next-best in 3-DFS (0.798) and the best in 5-DFS (0.933).

\section{Inconsistency assessment}

As shown in heat plots (Fig. 3), there is no evidence of inconsistency existing in the direct and indirect evidence in 3-OS, 5-OS, 3-DFS, and 5-DFS. Yet there was a sign of inconsistency regarding 5-OSR according to the results of heat plot.

\section{Subgroup analysis}

Among 28 included studies, 7 papers included only early-stage patients, 4 papers included both early-stage and locally advanced stages patients and 17 papers only included locally advanced stages patients (larynx stage III-IV and hypopharynx stage II-IV). For the treatments related to 7 papers included only early-stage patients were limited and the data were not sufficient enough to draw reliable conclusion, we decided to add a subgroup analysis of 17 papers only included locally advanced stages patients (larynx stage III-IV and hypopharynx stage II-IV). The network meta-analysis results were shown in Additional file 2: Table S2 and SUCRA results were shown in Additional file 3: Table S3. Surgery, RT, $\mathrm{RT}+\mathrm{S}$, ICRT, and CCRT were involved in subgroup analysis. In term of 3-OS and 5-OS, RT $+\mathrm{S}$ showed a relatively lower HR and better performance when compared with others without statistically significance; in term of 3-DFS and 5-DFS, CCRT and surgery alone had similar performance. In term of 5-OSR, RT $+S$ and surgery alone performed significantly better than RT (HR 0.39, 95\% CrI 0.17-0.9; HR 0.31, 95\% CrI 0.13-0.74). The SUCRA results shown in Additional file 3: Table S3 that $\mathrm{RT}+\mathrm{S}$ was the probably best treatments as for 3 -OS and 5-OS (SUCRA $=0.840,0.808$ ). CCRT may be the best treatments in increasing 3-DFS (0.695) while ICRT may be the best treatments in increasing 5 -DFS (0.771). Surgery alone ranked the highest in 5-OSR (0.337).

\section{Discussion}

Our NMA focused on comparative efficacies of six therapies of hypopharyngeal neoplasms and laryngeal neoplasms. Results of NMA indicated that surgery alone were significantly better than RT and CCRT according to 1-OS outcome. RT $+\mathrm{S}$ were significantly superior to RT, ICRT, and CCRT in terms of 3-OS. Meanwhile RT + S and surgery alone had significant advantage over RT in 5-OS. CCRT proved to be better than RT in 3-DFS. With regards to relative ranking analysis, $\mathrm{RT}+\mathrm{S}$ performed desirably in both OS and DFS while CCRT and ICRT were advantageous in DFS.

RT is utilized as the primary treatment for early laryngeal cancer in northern Europe and North America for its being an acknowledged treatment for selected laryngeal carcinomas that educes beneficial oncologic results [32]. For instance, RT could provide a possibility of curing un-resectable tumor. When compared with surgery, RT might provide less morbidity regarding subclinical disease and advantageous organ preservation [33].

Yet RT performed poorly in both OS and DFS according to our results, which is contrary to the results of a prior 
Table 2 Network meta-analysis results with hazard ratio (HR) for 3-OS, 5-OS, 3-DFS, and 5-DFS, and odds ratio (OR) for 5-OSR

\begin{tabular}{|c|c|c|c|c|c|}
\hline \multicolumn{6}{|l|}{$3-O S$} \\
\hline S & $0.8(0.64,1.00)$ & $0.95(0.95,1.68)$ & $1.04(0.83,0.30)$ & $0.85(0.65,1.11)$ & $0.81(0.65,1)$ \\
\hline $1.25(1.00,1.57)$ & RT & $1.19(0.68,2.08)$ & $1.31(1.06,1.61)$ & $1.07(0.84,1.36)$ & $1.02(0.83,1.24)$ \\
\hline $1.06(0.59,1.88)$ & $0.84(0.48,1.48)$ & TLM & $1.1(0.61,1.98)$ & $0.90(0.49,1.64)$ & $0.86(0.48,1.53)$ \\
\hline $0.96(0.77,1.20)$ & $0.77(0.62,0.94)$ & $0.91(0.51,1.63)$ & $\mathrm{RT}+\mathrm{S}$ & $0.82(0.68,0.98)$ & $0.78(0.64,0.94)$ \\
\hline $1.18(0.90,1.54)$ & $0.94(0.74,1.20)$ & $1.11(0.61,2.03)$ & $1.22(1.02,1.47)$ & ICRT & $0.95(0.76,1.20)$ \\
\hline $1.23(1.00,1.53)$ & $0.98(0.80,1.21)$ & $1.17(0.62,2.09)$ & $1.28(1.06,1.55)$ & $1.05(0.84,1.32)$ & CCRT \\
\hline \multicolumn{6}{|l|}{ 5-OS } \\
\hline S & $0.80(0.64,0.99)$ & $0.90(0.56,1.45)$ & $1.02(0.81,1.27$ & $0.91(0.69,1.21)$ & $0.86(0.68,1.10)$ \\
\hline $1.25(1.01,1.55)$ & RT & $1.13(0.71,1.80)$ & $1.27(1.01,1.59)$ & $1.14(0.87,1.48)$ & $1.08(0.86,1.35)$ \\
\hline $1.11(0.69,1.78)$ & $0.89(0.56,1.41)$ & TLM & $1.12(0.68,1.85)$ & $1.01(0.60,1.69$ & $0.96(0.58,1.58)$ \\
\hline $0.98(0.79,1.23)$ & $0.79(0.63,0.99)$ & $0.89(0.54,1.46)$ & $\mathrm{RT}+\mathrm{S}$ & $0.90(0.71,1.13)$ & $0.85(0.68,1.07)$ \\
\hline $1.10(0.83,1.46)$ & $0.88(0.68,1.15)$ & $0 . .99(0.59,1.67)$ & $1.12(0.89,1.40)$ & ICRT & $0.95(0.74,1.22)$ \\
\hline $1.16(0.91,1.47)$ & $0.93(0.74,1.16)$ & $1.05(0.63,1.72)$ & $1.18(0.93,1.48)$ & $1.05(0.82,1.35)$ & CCRT \\
\hline \multicolumn{6}{|l|}{ 3-DFS } \\
\hline S & $0.93(0.40,2.15)$ & $0.45(0.09,2.32)$ & $1.73(0.98,3.08)$ & $1.76(0.89,3.48)$ & $1.67(0.78,3.57)$ \\
\hline $1.08(0.46,2.49)$ & RT & $0.49(0.12,1.98)$ & $1.86(0.84,4.16)$ & $1.89(0.91,3.95)$ & $1.79(1.06,3.04)$ \\
\hline $2.21(0.43,11.29)$ & $2.05(0.51,8.31)$ & TLM & $3.82(0.76,19.20)$ & $3.88(0.80,18.84)$ & $3.68(0.82,16.42)$ \\
\hline $0.58(0.32,1.03)$ & $0.54(0.32,1.03)$ & $0.26(0.05,1.31)$ & $R T+S$ & $1.01(0.63,1.62)$ & $0.96(0.49,1.90)$ \\
\hline $0.57(0.29,1.13)$ & $0.53(0.25,1.1)$ & $0.26(0.05,1.25)$ & $0.99(0.62,1.58)$ & ICRT & $0.95(0.54,1.67)$ \\
\hline $0.6(0.28,1.28)$ & $0.56(0.33,0.94)$ & $0.27(0.06,1.21)$ & $1.04(0.53,2.05)$ & $1.05(0.60,1.86)$ & CCRT \\
\hline \multicolumn{6}{|l|}{ 5-DFS } \\
\hline S & $0.98(0.44,2.21)$ & $0.66(0.14,3.09)$ & $1.44(0.73,2.83)$ & $1.20(0.56,2.55)$ & $1.33(0.63,2.82)$ \\
\hline $1.02(0.45,2.28)$ & RT & $0.67(0.18,2.5)$ & $1.46(0.60,3.56)$ & $1.21(0.51,2.87)$ & $1.35(0.70,2.60)$ \\
\hline $1.51(0.32,7.09)$ & $1.49(0.40,5.55)$ & TLM & $2.18(0.45,10.67)$ & $1.81(0.38,8.7)$ & $2.01(0.46,8.74)$ \\
\hline $0.69(0.35,1.36)$ & $0.68(0.28,1.66)$ & $0.46(0.09,2.24)$ & $\mathrm{RT}+\mathrm{S}$ & $0.83(0.49,1.41)$ & $0.92(0.43,1.96)$ \\
\hline $0.84(0.39,1.78)$ & $0.82(0.35,1.94)$ & $0.55(0.11,2.66)$ & $1.21(0.71,2.05)$ & ICRT & $1.11(0.57,2.16)$ \\
\hline $0.75(0.35,1.60)$ & $0.74(0.38,1.43)$ & $0.5(0.11,2.16)$ & $1.08(0.51,2.30)$ & $0.9(0.46,1.74)$ & CCRT \\
\hline \multicolumn{6}{|l|}{$5-O S R$} \\
\hline S & $0.78(0.31,1.82)$ & $2.08(0.53,7.33)$ & $1.28(0.52,3.08)$ & $0.94(0.34,2.63)$ & $0.93(0.39,2.19)$ \\
\hline $1.29(0.55,3.24)$ & RT & $2.71(0.84,8.16)$ & $1.66(0.61,4.68)$ & $1.22(0.45,3.47)$ & $1.20(0.51,2.99)$ \\
\hline $0.48(0.14,1.87)$ & $0.37(0.12,1.20)$ & TLM & $0.61(0.15,2.79)$ & $0.45(0.11,2.14)$ & $0.44(0.12,1.84)$ \\
\hline $0.78(0.32,1.91)$ & $0.60(0.21,1.64)$ & $1.64(0.36,6.57)$ & $\mathrm{RT}+\mathrm{S}$ & $0.73(0.28,1.89)$ & $0.73(0.28,1.85)$ \\
\hline $1.06(0.38,2.97)$ & $0.82(0.29,2.24)$ & $2.24(0.47,9.15)$ & $1.37(0.53,3.55)$ & ICRT & $0.99(0.44,2.18)$ \\
\hline $1.07(0.46,2.57)$ & $0.83(0.33,1.95)$ & $2.25(0.54,8.40)$ & $1.38(0.54,3.57)$ & $1.01(0.46,2.28)$ & CCRT \\
\hline
\end{tabular}

Note: Figures in bold font show significant outcome

Abbreviation: $S$ surgery, $R T$ radiotherapy, $T L M$ transoral laser microsurgery, $R T+S$ surgery combined with radiotherapy, ICRT Induction chemotherapy radiotherapy, CCRT current chemotherapy radiotherapy, 3-OS 3-year overall survival, 5-OS 5-year overall survival, 3-DFS 3-year disease free survival, 5-DFS 5-year disease free survival;5-OSR, 5-year overall survival rate

NMA claiming that RT was associated with higher rates of OS and DFS than CT, CRT, and laryngectomy [32]. However, our results that CCRT and ICRT took advantageous over RT turned out to be consistent with paper conducted by Zackrisson et al., which concluded that CRT was better than RT [34]. Besides, TLM has been used in laryngeal malignant lesions since 1972 . As pointed out in prior NMAs, there are controversies in clinical literature regarding oncologic outcomes of TLM and RT for T1 glottis carcinoma, and plenty of comparisons between TLM and RT have been made. RT was inferior to TLM for T1 glottic carcinoma in OS according to Mo et al. [35], Zhong et al. [36], and Huang et al. [5]. Whereas Abdurehim et al. thought this question remained unanswered in systematic review since no significant difference was shown between RT and TLM [37], which is 

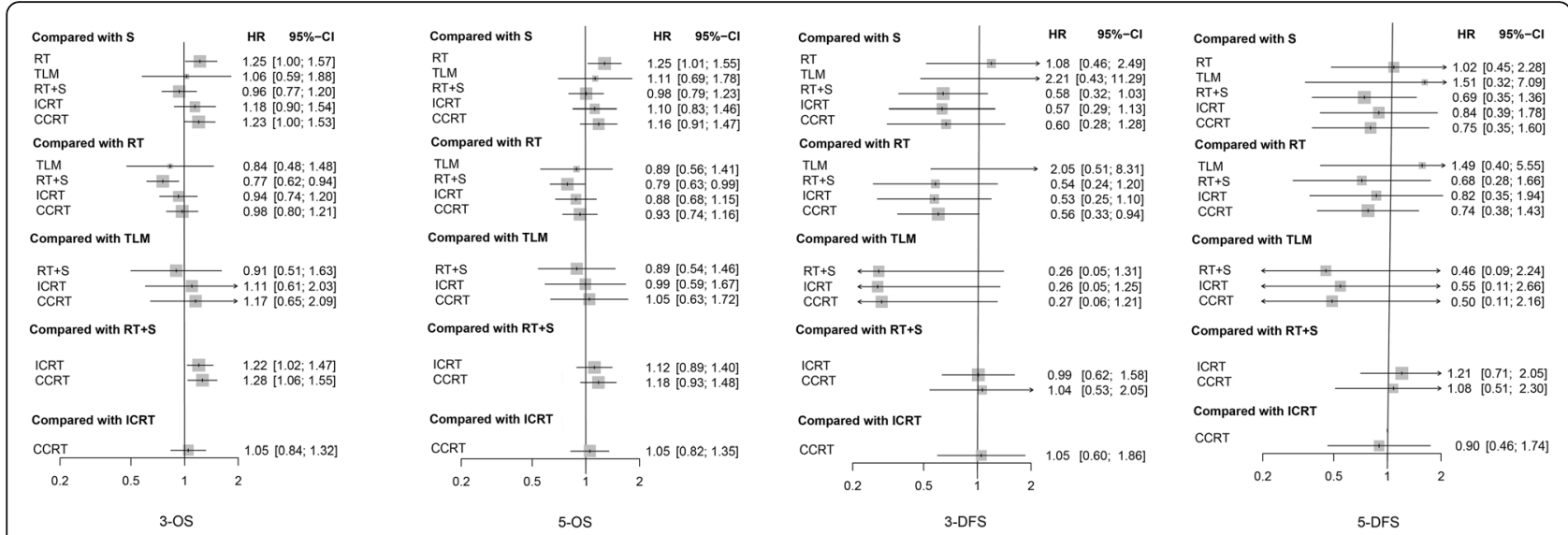

Fig. 2 Forest plots for four endpoints. Hazard ratio (HRs) and 95\% credible interval (Crls) indicate the relative efficacy under the corresponding endpoint. S, surgery; RT, radiotherapy; TLM, transoral laser microsurgery; RT + S, surgery combined with radiotherapy; ICRT, Induction chemotherapy radiotherapy; CCRT, current chemotherapy radiotherapy

also consistent with our results. More random-controlled and prospective trials should be conducted, based on which a more clinically reliable conclusion would be reached in future NMAs.

Furthermore, CCRT and ICRT demonstrated no significant difference on survival control in our analysis. Similar results were shown in $\mathrm{Ma}$ et al. paper which claimed that ICRT had no significant benefit compared to CCRT regarding OS or locoreginal control [38]. Yet Ma also claimed that ICRT generated desirable outcomes in larynx preservation [39]. More outcomes should be explored with regard to the effectiveness of ICRT and CCRT. The subgroup analysis of 17 studies reported patients with larynx stage III-IV and hypopharynx stage II-IV also showed similar results in term of 3-OS, 5-OS, 3-DFS, 5-DFS except that the results were all not statistically significant. More clinical researches will be required in the future.

There still exist several limitations in our NMA despite that we have conducted our work as meticulous as possible. First of all, there might be several deficiencies

Table 3 Surface under the cumulative ranking curve (SUCRA) for the treatments under five endpoints

\begin{tabular}{llllll}
\hline Treatments & 3-OS & 5-OS & 3-DFS & 5-DFS & 5-OSR \\
\hline S & 0.763 & 0.773 & 0.257 & 0.125 & 0.716 \\
RT & 0.243 & 0.151 & 0.291 & 0.457 & 0.076 \\
TLM & 0.582 & 0.486 & 0.088 & 0.244 & 0.687 \\
RT + S & 0.878 & 0.930 & 0.717 & 0.600 & 0.621 \\
ICRT & 0.244 & 0.248 & 0.846 & 0.639 & 0.413 \\
CCRT & 0.287 & 0.410 & 0.798 & 0.933 & 0.484
\end{tabular}

Abbreviation: $S$ surgery, $R T$ radiotherapy, $T L M$ transoral laser microsurgery, $R T$ $+S$ surgery combined with radiotherapy, ICRT Induction chemotherapy radiotherapy, CCRT current chemotherapy radiotherapy, 3-OS 3-year overall survival, 5-OS 5-year overall survival, 3-DFS 3-year disease free survival, 5-DFS 5 -year disease free survival, 5-OSR 5-year overall survival rate caused by the quality and non-unified standards of the included trials. For instance, patients' or surgeon's preference in these trials might lead to selection bias inevitably. Besides, a great proportion of the included trials were non-randomized or retrospective trials, which could increase heterogeneity of our NMA. Notably, toxicity of treatments analyzed in our NMA has not been investigated thoroughly because of a lack of data and comparable outcomes. It should not be ignored that a lack of full consideration on dose of RT and type of TLM might undermine the clinical value of our results to some extent. Secondly, we failed to take more combination therapies into consideration. Several trials and conventional meta-analyses have discovered an increase in efficacy of combination therapies such as chemotherapy combined with CCRT on survival control for some types of head and neck cancers [40-42]. Yet a comprehensive analysis regarding various combination treatments is hard to conduct on account of insufficient data in our included studies. Thirdly, sample sizes for some specific comparisons were not big enough. For instance, there were only 23 subjects treated by TLM in 3-DFS and 5-DFS. More prospective trials should be performed to reach more reliable conclusions and further studies focusing on combination therapies should be carried out. Fourthly, due to limited studies and insufficient data on early-stage patients, our analysis could not make a subgroup analysis of these patients. Therefore, future studies are needed to assess the impact of various treatments in the management of early-stage glottic larynx patients in order to provide more credible medical guideline.

\section{Conclusions}

RT combined with surgery turned out to be optimal therapy of all the outcomes while the efficacy of RT was 

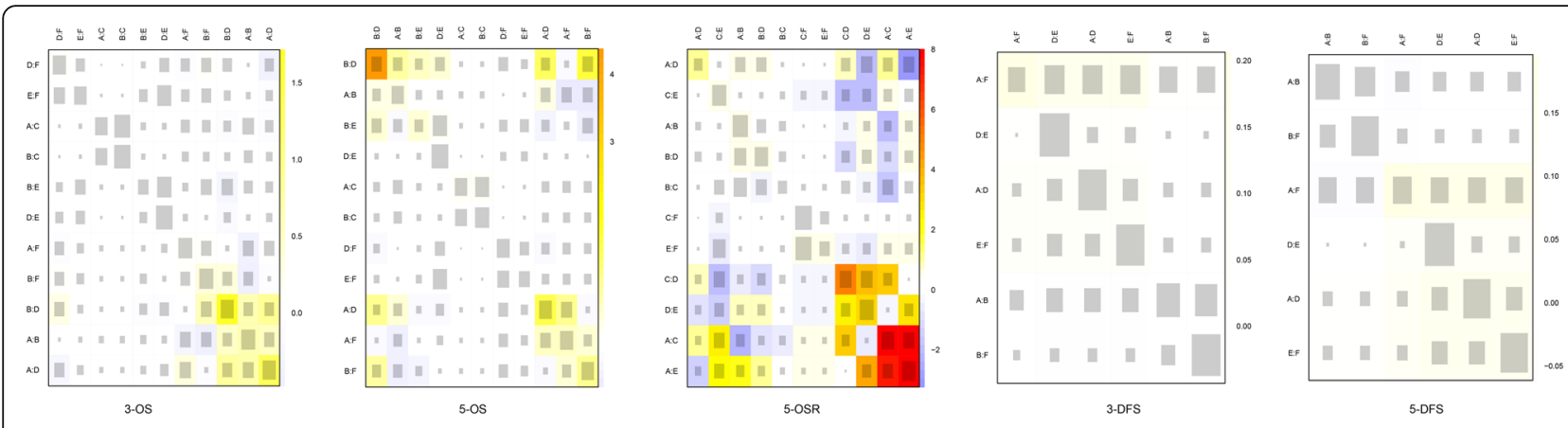

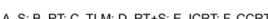

Fig. 3 The results of consistency analysis by heat plot for each outcome. The size of the gray squares indicates the contribution of the direct evidence (shown in the column) to the network evidence (shown in the row). The colors are associated with the change in inconsistency between direct and indirect evidence (shown in the row) and the warmer color represents the stronger inconsistency. S, surgery; RT, radiotherapy; TLM, transoral laser microsurgery; RT + S, surgery combined with radiotherapy; ICRT, Induction chemotherapy radiotherapy; CCRT, current chemotherapy radiotherapy

relatively poorer in the treatment of patients with larynx stage III-IV and hypopharynx stage II-IV. Also, the good performance of CCRT and ICRT in terms of DFS made them as secondary recommended therapies. There is no significant difference between surgery and TLM. The comparison of more related therapies will be better understood once results of more high-quality clinical trials conducted.

\section{Additional files}

Additional file 1: Table S1. Basic characteristics and endpoints of included studies. (DOCX $39 \mathrm{~kb}$ )

Additional file 2: Table S2. Network meta-analysis results of subgroup analysis for locally advanced hypopharyngeal and laryngeal neoplasms. (DOCX $19 \mathrm{~kb})$

Additional file 3: Table S3. SUCRA results of subgroup analysis for locally advanced hypopharyngeal and laryngeal neoplasms. (DOCX $16 \mathrm{~kb}$ )

\section{Abbreviations}

3/5-DFS: 3/5-year disease free survival; 3/5-OS: 3/5-year overall survival; 5OSR: 5-year overall survival rate; CCRT: Current chemoradiotherapy; Crls: Credible intervals; CRT: Chemoradiation therapy; HR: Hazard ratios; ICRT: Induction chemoradiotherapy; NMA: Network meta-analysis; PRISMA: Preferred reporting items for systematic reviews and meta-analyses; RT + S: Radiotherapy combined with surgery; RT: Radiotherapy; SUCRA: surface under the curve ranking area; TLM: transoral laser microsurgery

\section{Acknowledgments}

Not applicable

\section{Funding}

None.

\section{Availability of data and materials}

Not applicable.

\section{Authors' contributions}

JC (Che) made substantial contributions to conception and design. YW made acquisition of data. XZ and JC (Chen) made analysis and interpretation of data. JC (Che) have been involved in drafting the manuscript. YW, XZ and JC
(Chen) have been involved in revising it critically for important intellectual content. All authors have given final approval of the version to be published. All authors agreed to be accountable for all aspects of the work.

Ethics approval and consent to participate

Not applicable.

\section{Consent for publication}

Not applicable.

Competing interests

The authors declare that they have no competing interests.

\section{Publisher's Note}

Springer Nature remains neutral with regard to jurisdictional claims in published maps and institutional affiliations.

Received: 28 December 2017 Accepted: 27 February 2019

Published online: 29 March 2019

\section{References}

1. Marur S, Forastiere AA. Head and neck squamous cell carcinoma: update on epidemiology, diagnosis, and treatment. Mayo Clin Proc. 2016:91(3):386-96.

2. Forastiere AA, Zhang Q, Weber RS, Maor MH, Goepfert H, Pajak TF, Morrison W, Glisson B, Trotti A, Ridge JA, et al. Long-term results of RTOG 91-11: a comparison of three nonsurgical treatment strategies to preserve the larynx in patients with locally advanced larynx cancer. J Clin Oncol. 2013;31(7):845-52.

3. Wolf GT, Fisher SG, Hong WK, Hillman R, Spaulding M, Laramore GE, Endicott JW, McClatchey K, Henderson WG. Induction chemotherapy plus radiation compared with surgery plus radiation in patients with advanced laryngeal cancer. N Engl J Med. 1991;324(24):1685-90.

4. Ghadjar P, Simcock M, Zimmermann F, Betz M, Bodis S, Bernier J, Studer G, Aebersold DM, Swiss Group for Clinical Cancer R. Predictors of severe late radiotherapy-related toxicity after hyperfractionated radiotherapy with or without concomitant cisplatin in locally advanced head and neck cancer. Secondary retrospective analysis of a randomized phase III trial (SAKK 10/ 94). Radiother Oncol. 2012;104(2):213-8.

5. Huang G, Luo M, Zhang J, Liu H. Laser surgery versus radiotherapy for T1a glottic carcinoma: a meta-analysis of oncologic outcomes. Acta Otolaryngol. 2017;137(11):1204-9

6. Wolf GT, Bellile E, Eisbruch A, Urba S, Bradford CR, Peterson L, Prince ME, Teknos TN, Chepeha DB, Hogikyan ND, et al. Survival rates using individualized bioselection treatment methods in patients with advanced laryngeal Cancer. JAMA Otolaryngol Head Neck Surg. 2017;143(4):355-66.

7. Stokes WA, Jones BL, Bhatia S, Oweida AJ, Bowles DW, Raben D, Goddard $J A$, McDermott JD, Karam SD. A comparison of overall survival for patients 
with T4 larynx cancer treated with surgical versus organ-preservation approaches: a National Cancer Data Base analysis. Cancer. 2017;123(4):600-8.

8. Low TH, Yeh D, Zhang T, Araslanova R, Hammond JA, Palma D, Read N, Venkatesan V, MacNeil SD, Yoo J, et al. Evaluating organ preservation outcome as treatment endpoint for T1aN0 glottic cancer. Laryngoscope. 2017;127(6):1322-7.

9. Peng Z, Li Y, Jin L, Tao X, Cai X, Feng J, Liu R, Zhang Q, Li L. Retrospective analysis of therapeutic effect and prognostic factors on early glottic carcinoma. Photodiagn Photodyn Ther. 2016;15:167-71.

10. Marchiano E, Patel DM, Patel TD, Patel AA, Xue YE, Eloy JA, Baredes S, Park RC. Subglottic squamous cell carcinoma: a population-based study of 889 cases. Otolaryngol Head Neck Surg. 2016;154(2):315-21.

11. De Santis RJ, Poon I, Lee J, Karam I, Enepekides DJ, Higgins KM. Comparison of survival between radiation therapy and trans-oral laser microsurgery for early glottic cancer patients; a retrospective cohort study. J Otolaryngol Head Neck Surg = Le Journal d'oto-rhino-laryngologie et de chirurgie cervico-faciale. 2016;45(1):42

12. Timmermans AJ, de Gooijer CJ, Hamming-Vrieze O, Hilgers FJ, van den Brekel MW. T3-T4 laryngeal cancer in the Netherlands Cancer institute; 10year results of the consistent application of an organ-preserving/-sacrificing protocol. Head Neck. 2015;37(10):1495-503.

13. Timme DW, Jonnalagadda S, Patel R, Rao K, Robbins KT. Treatment selection for T3/T4a laryngeal Cancer: Chemoradiation versus primary surgery. Ann Otol Rhinol Laryngol. 2015;124(11):845-51.

14. Li P, Hu W, Zhu Y, Liu J. Treatment and predictive factors in patients with recurrent laryngeal carcinoma: a retrospective study. Oncol Lett. 2015;10(5): 3145-52.

15. Grover S, Swisher-McClure S, Mitra N, Li J, Cohen RB, Ahn PH, Lukens JN, Chalian AA, Weinstein GS, O'Malley BW Jr, et al. Total laryngectomy versus larynx preservation for T4a larynx Cancer: patterns of care and survival outcomes. Int J Radiat Oncol Biol Phys. 2015;92(3):594-601.

16. Hsin LJ, Fang TJ, Tsang NM, Chin SC, Yen TC, Li HY, Liao CT, Chen $\Vdash H$. Tumor volumetry as a prognostic factor in the management of T4a laryngeal cancer. Laryngoscope. 2014;124(5):1134-40.

17. Lefebvre JL, Pointreau Y, Rolland F, Alfonsi M, Baudoux A, Sire C, de Raucourt D, Malard O, Degardin M, Tuchais C, et al. Induction chemotherapy followed by either chemoradiotherapy or bioradiotherapy for larynx preservation: the TREMPLIN randomized phase II study. J Clin Oncol. 2013;31(7):853-9.

18. Lefebvre JL, Andry G, Chevalier D, Luboinski B, Collette L, Traissac L, de Raucourt D, Langendijk JA. Laryngeal preservation with induction chemotherapy for hypopharyngeal squamous cell carcinoma: 10-year results of EORTC trial 24891. Ann Oncol. 2012;23(10):2708-14.

19. Patel UA, Howell LK. Local response to chemoradiation in T4 larynx cancer with cartilage invasion. Laryngoscope. 2011;121(1):106-10.

20. Mahler V, Boysen M, Brondbo K. Radiotherapy or CO (2) laser surgery as treatment of T (1a) glottic carcinoma? Eur Arch Otorhinolaryngol. 2010; 267(5):743-50

21. Dinapoli N, Parrilla C, Galli J, Autorino R, Micciche F, Bussu F, Balducci M, D'Alatri L, Marchese R, Rigante M, et al. Multidisciplinary approach in the treatment of $\mathrm{T} 1$ glottic cancer. The role of patient preference in a homogenous patient population. Strahlenther Onkolo. 2010;186(11):607-13.

22. Schrijvers ML, van Riel EL, Langendijk JA, Dikkers FG, Schuuring E, van der Wal JE, van der Laan BF. Higher laryngeal preservation rate after CO2 laser surgery compared with radiotherapy in T1a glottic laryngeal carcinoma. Head Neck. 2009;31(6):759-64.

23. Thurnher D, Erovic BM, Frommlet F, Brannath W, Ehrenberger K, Jansen B, Selzer E, Grasl MC. Challenging a dogma--surgery yields superior long-term results for T1a squamous cell carcinoma of the glottic larynx compared to radiotherapy. Eur J Surg Oncol. 2008;34(6):692-8.

24. Boscolo-Rizzo P, Maronato F, Marchiori C, Gava A, Da Mosto MC. Long-term quality of life after total laryngectomy and postoperative radiotherapy versus concurrent chemoradiotherapy for laryngeal preservation. Laryngoscope. 2008;118(2):300-6.

25. Andreadis C, Iliopoulou C, Sidiras T, Boutis A, Diamantopoulos N, Vahtsevanos K, Gennatas K, Mouratidou D. Neoadjuvant chemotherapy followed by radiotherapy versus concurrent chemoradiotherapy for larynx preservation in patients with advanced laryngeal cancer. J BUON. 2007;12(3):341-7.

26. Bensadoun RJ, Benezery K, Dassonville O, Magne N, Poissonnet G, Ramaioli A, Lemanski C, Bourdin S, Tortochaux J, Peyrade F, et al. French multicenter phase III randomized study testing concurrent twice-a-day radiotherapy and cisplatin/5-fluorouracil chemotherapy (BiRCF) in unresectable pharyngeal carcinoma: results at 2 years (FNCLCC-GORTEC). Int J Radiat Oncol Biol Phys. 2006:64(4):983-94.

27. Richard JM, Sancho-Garnier H, Pessey JJ, Luboinski B, Lefebvre JL, Dehesdin D, Stromboni-Luboinski M, Hill C. Randomized trial of induction chemotherapy in larynx carcinoma. Oral Oncol. 1998;34(3):224-8.

28. Beauvillain C, Mahe M, Bourdin S, Peuvrel P, Bergerot P, Riviere A, Vignoud J, Deraucourt D, Wesoluch $M$. Final results of a randomized trial comparing chemotherapy plus radiotherapy with chemotherapy plus surgery plus radiotherapy in locally advanced resectable hypopharyngeal carcinomas. Laryngoscope. 1997;107(5):648-53.

29. Bryant GP, Poulsen MG, Tripcony L, Dickie GJ. Treatment decisions in T3NOMO glottic carcinoma. Int J Radiat Oncol Biol Phys. 1995;31(2):285-93.

30. Frank JL, Garb JL, Kay S, McClish DK, Bethke KP, Lind DS, Mellis M, Slomka W, Sismanis A, Neifeld JP. Postoperative radiotherapy improves survival in squamous cell carcinoma of the hypopharynx. Am J Surg. 1994; 168(5):476-80.

31. Jones AS, Cook JA, Phillips DE, Soler Lluch E. Treatment of T3 carcinoma of the larynx by surgery or radiotherapy. Clin Otolaryngol Allied Sci. 1992;17(5): $433-6$.

32. Luo XN, Chen LS, Zhang SY, Lu ZM, Huang Y. Effectiveness of chemotherapy and radiotherapy for laryngeal preservation in advanced laryngeal cancer: a meta-analysis and systematic review. Radiol Med. 2015; 120(12):1153-69.

33. Feng $Y$, Wang $B$, Wen $S$. Laser surgery versus radiotherapy for T1-T2N0 glottic cancer: a meta-analysis. ORL J Otorhinolaryngol Relat Spec. 2011; 73(6):336-42.

34. Zackrisson B, Mercke C, Strander H, Wennerberg J, Cavallin-Stahl E. A systematic overview of radiation therapy effects in head and neck cancer. Acta Oncol. 2003;42(5-6):443-61.

35. Mo HL, Li J, Yang X, Zhang F, Xiong JW, Yang ZL, Tan J, Li B. Transoral laser microsurgery versus radiotherapy for $\mathrm{T} 1$ glottic carcinoma: a systematic review and meta-analysis. Lasers Med Sci. 2017;32(2):461-7.

36. Zhong A, Xu X, Fan H, Wang L, Niu Y. Transoral laser microsurgery for recurrent laryngeal carcinoma after primary treatment: a systematic review and meta-analysis. J Cancer Res Ther. 2015;11(Suppl 2):C173-8.

37. Abdurehim Y, Hua Z, Yasin Y, Xukurhan A, Imam I, Yuqin F. Transoral laser surgery versus radiotherapy: systematic review and meta-analysis for treatment options of T1a glottic cancer. Head and neck. 2012;34(1):23-33.

38. Ma J, Liu Y, Yang X, Zhang CP, Zhang ZY, Zhong LP. Induction chemotherapy in patients with resectable head and neck squamous cell carcinoma: a meta-analysis. World J Surg Oncol. 2013;11:67.

39. Ma J, Liu Y, Huang XL, Zhang ZY, Myers JN, Neskey DM, Zhong LP. Induction chemotherapy decreases the rate of distant metastasis in patients with head and neck squamous cell carcinoma but does not improve survival or locoregional control: a meta-analysis. Oral Oncol. 2012;48(11): 1076-84.

40. Saitoh Jl, Shirai K, Imaeda M, Musha A, Abe T, Shino M, Takayasu Y, Takahashi K, Chikamatsu K, Nakano T. Concurrent chemoradiotherapy with conventional fractionated radiotherapy and low-dose daily cisplatin plus weekly docetaxel for T2N0 glottic cancer. Radiat Oncol. 2017;12(1):39.

41. Yuan $\mathrm{C}, \mathrm{Xu} \mathrm{XH}$, Chen Z. Combination treatment with antiEGFR monoclonal antibodies in advanced nasopharyngeal carcinoma : a meta-analysis. J BUON. 2015;20(6):1510-7.

42. Vanderveken OM, Szturz P, Specenier P, Merlano MC, Benasso M, Van Gestel D, Wouters K, Van Laer C, Van den Weyngaert D, Peeters M, et al. Gemcitabine-based Chemoradiation in the treatment of locally advanced head and neck Cancer: systematic review of literature and meta-analysis. Oncologist. 2016;21(1):59-71. 